\title{
Fall Detection Application Using Kinect
}

\author{
Kartika Gunadi, Liliana, Jonathan Tjitrokusmo \\ Department of Informatics \\ Petra Christian University \\ Surabaya, Indonesia \\ kgunadi@petra.ac.id, lilian@ petra.ac.id, m26413041@john.petra.ac.id
}

\begin{abstract}
This paper describes method for fall detection, because fall is a serious problem and often resulting to injury, which can endanger the safety of the person. Therefore, falling detection is crucially needed. The device being used is Kinect, which also could be used to detect people. The detection is using Microsoft Kinect SDK's assistance. Kinect can detect a person in front of it and processing it to create a skeleton of the person. The method being used is to get a set of data on the position of the person. Next, the rate of change in position would be calculated with the available formulae. The data obtained would be selected, in order to distinguish the activities undertaken. When fall is detected, the application can provide the alert.
\end{abstract}

Keywords-Kinect; Microsoft Kinect SDK; skeleton; fall; fall detection.

\section{INTRODUCTION}

Falling is not something new, but still a lot of people underestimate it. Fall can be interpreted as a movement which inadvertently makes the body down towards the floor or some of the lower levels [1]. This falls usually lasts for one to two seconds [2]. According to the Centers for Disease Control and Prevention, one in five people fall causing serious injuries such as broken bones or head injuries and falls are the main cause of death for the elderly [3]. Intensively watching out the elderly is needed, where one of three elderly falls annually. Though, falls are more common in children, but compared to them, the elderly who fall, ten times more likely to receive treatment in hospital and eight times more likely to die [4]. So, fall should not be underestimated, although not all falls would be fatal, giving first aid is very important [5].

\section{LITERATURE}

This application that had been made, utilizes Kinect to detect the person. Kinect is one of Microsoft's Xbox 360 product that is used to allow users to control and interact with the console. Kinect uses RGB-D camera, which can capture RGB images like a regular camera and can capture the depth or distance of the person. Depth capture is an excess of Kinect and by capturing Depth, then the received image has a clear position. The referred position is the location of the person in the $\mathrm{X}$-axis, $\mathrm{Y}$-axis, and $\mathrm{Z}$ axis. The minimum distance for people to be detected is 0.6 meters and the maximum distance reached 4 to 5 meters, and at 30 frames per second [5]. In order to process the data in Kinect, Kinect SDK is required. The processed data includes the performing calculations and processing on the $\mathrm{X}$ axis, $\mathrm{Y}$ axis, and $\mathrm{Z}$ axis that has been captured by Kinect.

Software Development Kit (SDK) is a set of software development tools used to develop or create applications for a certain software package. Microsoft Kinect SDK is used to acquire and process data on the Kinect. This SDK allows to access the raw data from the camera sensor of Kinect. Also, there is Skeletal Tracking which has the ability to detect people moving in the area of detection Kinect. By using skeletal tracking, the information that obtained will be much beneficial for the algorithms which associated with the skeleton, such as accessing data on the position of the head, shoulders, spine, hips and can process [5].

\section{METHODS}

Fall can be detected by calculating the speed of change position of the person on the environment [6]. Therefore, it can be calculated against the person's position. The position of skeleton that has been obtained, is being expounded in the value of the $\mathrm{X}$-axis, $\mathrm{Y}$-axis and $\mathrm{Z}$-axis so it can calculate the width, height and depth of the person. Calculate the width, height and depth by using Equation 1, Equation 2 and Equation 3 [7]:

$$
\begin{aligned}
& \text { Width }=|X \min -X \max | \\
& \text { Height }=|Y \min -Y \max | \\
& \text { Depth }=|Z \min -Z \max |
\end{aligned}
$$

People who fall will experience changes in height, width or distance. Usually the height of the person will be drastically decreased while the accretion of width or distance would happen, depending on the direction of the fall. Therefore, the calculation of the speed of changes in height and width or depth is required.

Calculating the speed of changes in height can be started by calculating the height of the person in each frame. After that, calculate the speed of changes in height of each frame by dividing the height with the time of each frame. The formulation is shown in Equation 4 [8]:

$$
\text { whead }=\frac{\operatorname{head}(k)-\operatorname{head}(k-1)}{\mathrm{t}(\mathrm{k})-\mathrm{t}(\mathrm{k}-1)}
$$

vhead $=$ speed of changes in height

head $(\mathrm{k})=$ height in frame $(\mathrm{k})$

$\mathrm{t}(\mathrm{k}) \quad=$ time in frame $(\mathrm{k})$. 
After getting the height-speed changes from the width and distance data that have been owned, the speed of changes in width and distance would be found as well. Because the increased of distance or width has not been known yet, compounding the distance and width data would help to discover them. It can be formulated as in Equation 5:

$$
L=\sqrt{J^{2}+L^{2}}
$$

$\mathrm{J}=$ depth

$\mathrm{L}=$ width.

$\mathrm{JL}$ from the data is used to find the speed of changes in width and distance with JL divided by the time of each frame. It can be formulated as in Equation 6:

$$
V j l=\frac{j l(\hat{i})-j l(i-1)}{t(\hat{i})-t(\hat{i}-1)}
$$

$\mathrm{V}_{\mathrm{JL}}=$ speed of change in $\mathrm{JL}$

$\mathrm{JL}_{\mathrm{i}}=\mathrm{JL}$ in frame (i)

$\mathrm{t}_{\mathrm{i}}=$ time in frame (i).

From the height-speed changes data and speed changes in distance and width, it can be seen if there is conspicuous speed happened. In order to ensure the occurrence of conspicuous speed, a reference value has determined. If the height-speed changes, the distance and the width of that person exceed the reference value for the speed of changes in height, distance and width that have been determined, then most likely fall happened, however, the possibility of not falling can also happen when the person is sitting, exercising or doing other things. So a further confirmation would be done by calculating the position of the head, hands, and feet and the speed of changes in position.

Speed of changes in head position can be found from the formulae in Equation 7:

$$
\text { W head }=\frac{\text { head (i) }- \text { head }(i-1)}{t(i)-t(i-1)}
$$

$\mathrm{v}$ head $=$ speed of changes in head position

head(i) $=$ the position of head at frame(i)

$\mathrm{t}(\mathrm{i})=$ time in frame (i).

Speed of changes in hands and feet can be discovered from the formulas in Equation 8 and Equation 9:

$$
\text { (1) hand }=\frac{\text { hand }(i)-\text { hand }(i-1)}{t(j)-t[i-1]}
$$

$\mathrm{v}$ hand $=$ speed of changes in hands position

hand(i) $=$ the position of hands at frame (i)

$\mathrm{t}(\mathrm{i}) \quad=$ time in frame (i).

$$
\text { (1) } \operatorname{leg}=\frac{\operatorname{leg}(i)-\operatorname{leg}(i-1)}{t(i)-t[i-1]}
$$

$\mathrm{v}$ leg $=$ speed of changes in leg position

$\operatorname{leg}(\mathrm{i})=$ the position of leg at frame (i)

$\mathrm{t}$ (i) = time in frame (i).

Both of left and right hands as well as left and right leg are used to calculate the speed of changes in hands and feet.

After having the speed of changes in the position of the head, hands and feet, the data is compared against a reference value of the speed of changes in the position of the head, hands, and feet. If the speed of changes in these positions exceeds the reference value, it can be concluded that the person falling. To better explain it can use flowchart in Fig. 1.

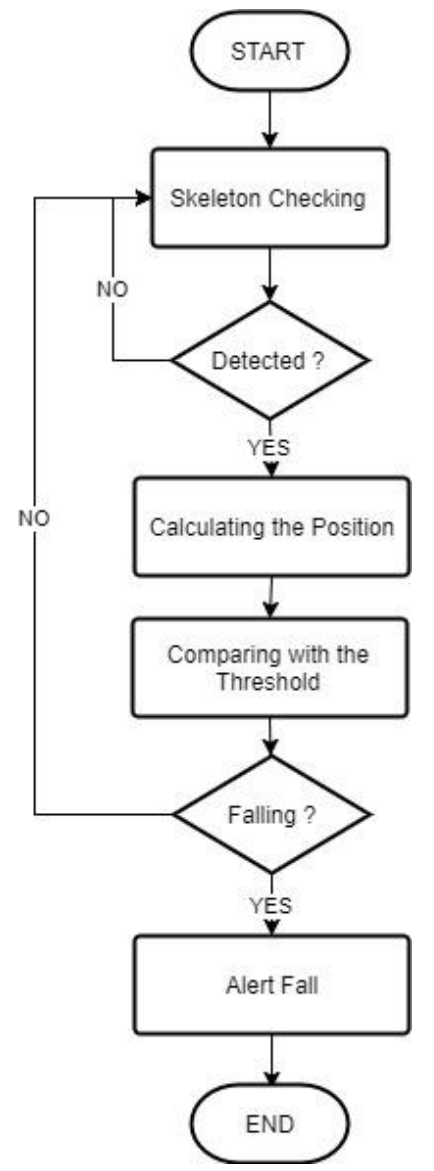

Figure 1. Flowchart of the application

In the beginning, there would be a checking prior to the Kinect, then checking the skeleton that have been detected. If they have been detected, the next process is to calculating the position, such as height, width, depth, joint head, hands, and legs. From the obtained data of position, the speed of changes in position would be calculated with the previous equation. The results would be compared with the reference values that have been determined before. If the value exceeds these limits, it can be concluded that fall occurred. When it happens, the application will send an alert or warning.

\section{IMPLEMENTATION}

This application requires two software supports, which are WPF .Net in VB language and the Microsoft Kinect SDK. The selection of the two software is affected by the supports of WPF .Net towards the use of Microsoft Kinect SDK, which enables to process the image results of Kinect.

Skeleton is a very important factor in these applications, where fall checking is based on the obtained data of the skeleton. The skeleton contains the data position of each joint that has been portrayed on screen. Illustration of the skeleton that is detected can be seen in Fig. 2. 


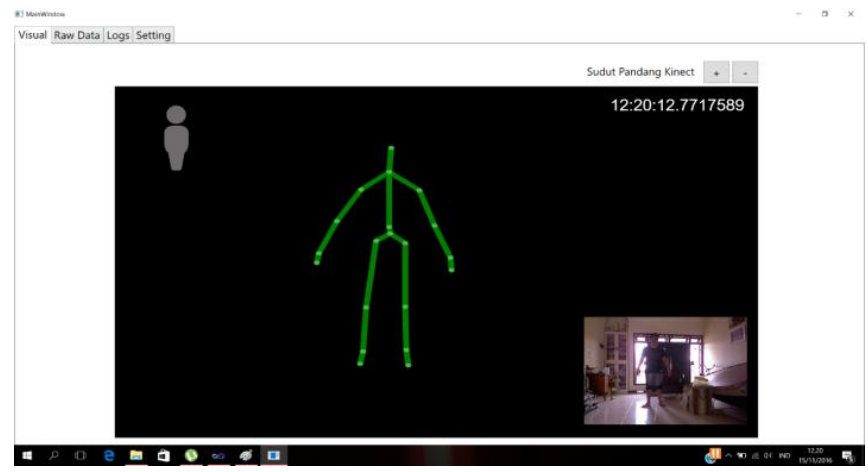

Figure 2. Skeleton that formed

TABLE I. FAll Test PRoducing Vh, VJL AND VheAD

\begin{tabular}{cccc}
\hline Frame \# & Vh & Vjl & Vhead \\
\hline 1 & -3 & -6 & 4 \\
2 & -4 & -5 & 5 \\
3 & -3 & 2 & 6 \\
4 & -3 & -2 & 7 \\
5 & -5 & 4 & 10 \\
6 & -7 & 6 & 12 \\
7 & -6 & 7 & 15 \\
8 & -6 & 9 & 17 \\
9 & -5 & 10 & 19 \\
10 & -4 & 9 & 21 \\
11 & -3 & 6 & 23 \\
12 & -4 & 8 & 26 \\
13 & -10 & 14 & 27 \\
14 & -11 & 15 & 29 \\
15 & -11 & 24 & 31 \\
16 & -23 & 32 & 31 \\
17 & -41 & 29 & 33 \\
18 & -50 & 35 & 58 \\
19 & -47 & 26 & 59 \\
20 & -53 & 40 & 61 \\
21 & -50 & 37 & 57 \\
22 & -53 & 42 & 55 \\
23 & -56 & 18 & 57 \\
24 & -56 & 50 & 56 \\
25 & -59 & 48 & 54 \\
26 & -59 & 33 & 52 \\
27 & -61 & 50 & 50 \\
28 & -60 & 48 & 47 \\
29 & -52 & 44 & 45 \\
30 & -52 & 45 & 44 \\
\hline & & &
\end{tabular}

The extracted data from the skeleton would be used to checking the fall. The processed data that has been detected is the height, width, depth, vh, vjl, yhead, yrighthand, ylefthand, yrightleg, yleftleg, vhead, vrighthand, vlefthand, vrightleg and vleftleg.

Height is the overall height of the person. From the height, vhead can be found, which interprets as the speed of changes in height. Width is the width of skeleton based on the x-axis. Depth is depth or depth of the skeleton based on the $\mathrm{z}$ axis. The depth and width can attain $\mathrm{jl}$, which is the compounding of depth and width. After getting jl, vjl can be obtained as it is the speed of changes in width-depth. yhead is the position of the head on the y-axis. yhead is used to find
TABLE II. FALL TEST DATA THAT GENERATE V LEFT HAND, V RIGHT HAND, V LEFT LEG AND V RIGHT LEG

\begin{tabular}{|c|c|c|c|c|}
\hline Frame\# & V LeftHand & V RightHand & V LeftLeg & V RightLeg \\
\hline 1 & 6 & 5 & 5 & 0 \\
\hline 2 & 6 & 4 & 6 & 0 \\
\hline 3 & 8 & 5 & 1 & 2 \\
\hline 4 & 5 & 7 & 4 & 2 \\
\hline 5 & 6 & 7 & 2 & 4 \\
\hline 6 & 7 & 8 & 4 & 5 \\
\hline 7 & 8 & 8 & 5 & 9 \\
\hline 8 & 8 & 8 & 7 & 11 \\
\hline 9 & 8 & 7 & 7 & 13 \\
\hline 10 & 8 & 6 & 12 & 18 \\
\hline 11 & 8 & 5 & 14 & 19 \\
\hline 12 & 9 & 7 & 19 & 22 \\
\hline 13 & 10 & 10 & 3 & 18 \\
\hline 14 & 12 & 12 & 2 & 21 \\
\hline 15 & 13 & 14 & 9 & 22 \\
\hline 16 & 15 & 18 & 10 & 3 \\
\hline 17 & 17 & 22 & 16 & 6 \\
\hline 18 & 22 & 12 & 19 & 2 \\
\hline 19 & 20 & 12 & 22 & 2 \\
\hline 20 & 20 & 10 & 17 & 0 \\
\hline 21 & 20 & 19 & 20 & 2 \\
\hline 22 & 14 & 18 & 15 & 6 \\
\hline 23 & 9 & 15 & 23 & 8 \\
\hline 24 & 1 & 20 & 15 & 16 \\
\hline 25 & 0 & 20 & 24 & 16 \\
\hline 26 & 1 & 21 & 27 & 22 \\
\hline 27 & 1 & 21 & 28 & 25 \\
\hline 28 & 3 & 19 & 34 & 28 \\
\hline 29 & 5 & 17 & 8 & 30 \\
\hline 30 & 7 & 14 & 7 & 31 \\
\hline
\end{tabular}

vhead or the speed of changes in head position on the y-axis. ylefthand, yrighthand, yleftleg and yrightleg are the position of the left hand, right hand, left leg and right leg on the yaxis. After speed of changes in the y-axis position has been gotten, vlefthand, vrighthand, vleftleg and vrightleg would be found as well.

Falling has initial position with a standing start position and finish up with the laying on the floor position [8]. The results of the fall can be seen in Table I for the frame number, vh, vjl, and vheads (VHead). Frame number is the number of recording data. Each frame consists 0,037 milliseconds of time. For vleft hand (VLefthand), vrighthand (VRighthand), vleftleg (VLeftleg), and vrightleg (VRightleg) can be seen in Table II, with a similar falling activity to Table I.

\section{CONCLUSION}

When there is fall, the height, width, and depth would likely to change. The height will decrease but the width and depth will accumulate because it becomes $\mathrm{jl}$, vhead that has been found, will have a large negative value, due to the radical changes of height in a short time. Vjl will also have a large value, because of the accretion of width and depth. The speed of changes in head also has a large value, which even greater than vhead. For the speed of changes in the position of hands, the left hand and the right hand are not necessarily 
have the same value, though it is in the same frame. Both left and right hands are more likely to equally experience the rapid change of position, yet the value is not as big as vhead. The speed of changes in feet also has a similar value to the speed of changes in the position of hands speed in change position and still, not as big as $\mathrm{v}$ head.

\section{REFERENCES}

[1] S.S. Arote and R.S. Bhosale, "Fall Detection System Using Accelerometer Principals with Arduino Development Board," Int. J. Advance Research in Computer Science and Management Studies, vol. 3, no. 9, pp. 289-293, Sep. 2015.

[2] X. Yu, "Approaches and Principles of Fall Detection for Elderly and Patient," Proc. 10th Int. Conf. on E-health Networking, Applications and Services (HealthCom), Singapore, Jul. 2008, pp. 42-47, doi: 10.1109/HEALTH.2008.4600107.

[3] CDC, "Important Facts about Falls," US Centers for Disease Control and Prevention. [Online] Available: http://www.cdc.gov/ HomeandRecreationalSafety/Falls/adultfalls.html.

[4] G.F. Fuller, "Falls in the Elderly," American Family Physician, vol. 61, no. 7, pp. 2159-2168, Apr. 2000. [Online] Available: http://www.aafp.org/afp/2000/0401/p2159.html
[5] B. Kwolek and M. Kepski, "Fall Detection Using Kinect Sensor and Fall Energy Image," Proc. Int. Conf. on Hybrid Artificial Intelligence Systems (HAIS), Salamanca (Spain), Sep. 2013, Springer LNCS, vol. 8073, J.S. Pan, M.M. Polycarpou, M. Woźniak, A.C.P.L.F. de Carvalho, H. Quintián, and E. Corchado, Eds., pp. 294-303, doi: 10.1007/978-3-642-40846-5_30.

[6] C. Kawatsu, J. Li, and C.J. Chung, "Development of a Fall Detection System with Microsoft Kinect," Proc. 1st Int. Conf. on Robot Intelligence Technology and Applications (RiTA), Gwangju (South Korea), Dec. 2012, Springer AISC, vol. 208, J.H. Kim, E.T. Matson, H. Myung, and P. Xu, Eds., pp. 623-630, doi: 10.1007/978-3-64237374-9.

[7] G. Mastorakis and D. Makris, "Fall Detection System Using Kinect's Infrared Sensor," J. Real-Time Image Processing, vol. 9, no. 4, pp. 635-646, Dec. 2014, doi: 10.1007/s11554-012-0246-9.

[8] V. Bevilacqua, N. Nuzzolese, D. Barone, M. Pantaleo, M. Suma, D. D'Ambruoso, A. Volpe, C. Loconsole, and F. Stroppa, "Fall Detection in Indoor Environment with Kinect Sensor," Proc. IEEE Int. Symp. on Innovations in Intelligent Systems and Applications (INISTA), Alberobello (Italy), Jun. 2014, pp. 319-324, doi: 10.1109/INISTA.2014.6873638 